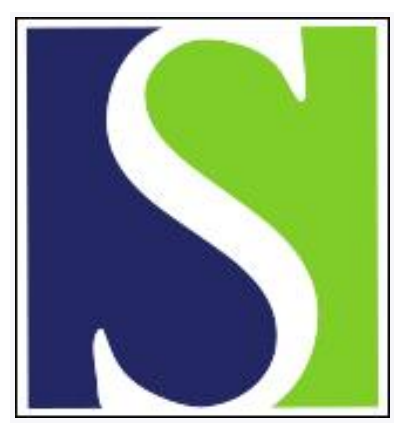

Scand J Work Environ Health 1990;16(4):293

https://doi.org/10.5271/sjweh.1792

Issue date: 01 Aug 1990

Directionality in the association between lumbar degenerative changes and back pain.

by Rossignol $\mathrm{M}$

This article in PubMed: www.ncbi.nlm.nih.gov/pubmed/2143846

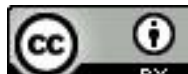


Scand J Work Environ Health 1990;16:293

\section{Directionality in the association between lumbar degenerative changes and back pain}

I would like to comment on the important paper recently published by Riihimäki et al ("Radiographically Detectable Lumbar Degenerative Changes as Risk Indicators of Back Pain: A Cross-Sectional Epidemiologic Study of Concrete Reinforcement Workers and House Painters." Scand J Work Environ Health 1989;15:280-5). The authors found and described an association of moderate to severe degenerative changes (in lumbar radiographs) with an increased risk of sciatic pain but not with lumbago or nonspecific back pain. Whereas the cross-sectional nature of the study did not allow the determination of which (the radiographic change or the pain) occurred first, the pathophysiology of sciatic pain supports the logic that pain can follow degenerative changes in this particular case.

The authors deemed "reasonable to study the role of lumbar degeneration as a predictor for recent symptoms [in the past twelve months] because degeneration is a slowly advancing process [p 284]." The use of the term "predictor" implies, in this case, a directionality. There is enough evidence in the literature to prove that back symptoms also develop over a long period of time and therefore can precede rather than follow radiographically detectable degenerative changes. In fact, a history of back pain is one of the best predictors of recurring back symptoms (1). In effect the authors also found that workers with an earlier history of back accident had over twice the risk of having sciatic pain in the past 12 months, a finding accompanied by a caution indicating potential recall bias but consistent with current scientific knowledge.

The regression analysis was performed with the assumption that symptoms of pain were the "effect" (dependent variable) and radiographic changes were one of the "explanatory variables." If indeed symptoms of sciatica are associated with a previous history of back symptoms (called "back accidents" by Riihimäki et al), it would be equally valid to inverse the roles in the regression and study the predictive value of pain for lumbar degenerative changes.

Finally, the authors rightly conclude that the study did not support the use of spinal radiographs as a predictor of back pain in preemployment screening procedures. However the paper created much interest among occupational physicians because it found an association that has been rejected until now, and it proposed, through its analysis, a directionality assigning a cause (radiographic change) and an effect (back pain) which was only assumed for analytical purposes and could as easily be turned around. The directionality can only be determined in a longitudinal design.

\section{References}

1. Yu T, Roth LH, Wise RA, Kilian JD, Weir FW. Low back pain in industry: an old problem revisited. J Occup Med 1984;26:517-24.

Michel Rossignol, MD, DSC

Department of Community Health

Sacre-Coeur Hospital

5945 Gouin Blvd W, suite 200

Montreal, Quebec, Canada H4J 1E5

\section{Authors' reply}

We would like to thank Dr Rossignol for his interest in our paper. We agree with his comment about the directionality in the association between lumbar degenerative changes and back pain. Indeed, from clinical experience, it is well established that symptoms of sciatica may occur before disc degeneration is advanced enough to be visualized in a plain lumbar radiograph. We also found lumbar degenerative changes to be a significant explanatory factor for sciatic pain retrospectively (Scand J Work Environ Health 1989;15:280285, Scand J Work Environ Health 1989;15:415-423) but not prospectively in a five-year follow-up (Scand $J$ Work Environ Health 1989;15:415-423). As Dr Rossignol points out, the directionality can only be determined with a longitudinal study design. Repeated radiographic examinations would be needed along with repeated symptom surveys. Today magnetic resonance imaging would be the technique of choice because it does not have any known adverse health effects.

On behalf of the team,

Hilkka Riihimäki, MD, MSc

Institute of Occupational Health

Topeliuksenkatu 41 a A

SF-00250 Helsinki Finland 